\title{
Should the esophageal cancer staging system be revised?
}

Valerie W. Rusch, MD



See related article on page 1103.

\footnotetext{
From the Thoracic Service, Department of Surgery, Memorial Sloan-Kettering Cancer Center, New York, NY

Received for publication Sept 20, 2002; accepted for publication Oct 21, 2002.

Address for reprints: Valerie W. Rusch, MD, Chief, Thoracic Service, Department of Surgery, Memorial Sloan-Kettering Cancer Center, 1275 York Ave, New York, NY 10021 (E-mail: ruschv@mskcc.org).

J Thorac Cardiovasc Surg 2003;125:992-3

Copyright ( $\odot 2003$ by The American Association for Thoracic Surgery

$0022-5223 / 2003 \$ 30.00+0$

doi: $10.1067 / \mathrm{mtc} .2003 .259$
}



ccurate TNM staging plays a pivotal role in cancer management and research. The TNM staging system for esophageal cancer is frequently viewed with discontent by thoracic surgeons. Yet, the sixth editions of the American Joint Committee on Cancer (AJCC) and the Union Internationale Contre le Cancer (UICC) staging manuals, published in 2002, offered minimal changes in the chapter on esophageal staging. ${ }^{1,2}$ The AJCC chapter notes the remarkable epidemiologic shift that has occurred in this disease with a rapid increase in the number of adenocarcinomas of the distal esophagus and gastroesophageal junction. The location of the lymph nodes involved by gastroesophageal junction tumors was added to the section on nodal descriptors, and a lymph node map previously proposed by Casson and colleagues ${ }^{3}$ was added to encourage uniform nomenclature of lymph nodes removed at surgery. However, the TNM descriptors and stage classifications were not changed from the previous editions of the staging manuals.

The lack of change in the esophageal staging system frustrates many surgeons who believe that it does not stratify patients appropriately for prognosis. However, a staging system must be evidence-based, prognostically accurate, easy to use, and internationally accepted. The AJCC task force for the chapters on lung and esophageal cancer staging that I chaired ${ }^{1}$ strongly considered changing the staging system for esophageal cancer, but in conjunction with the editors of the manual ultimately did not do so because, unfortunately, there was insufficient published data to support a proposal for a new system. In addition, the AJCC and the UICC coordinate acceptance of changes in staging systems to maintain international concordance. Proposed changes must be based on data considered persuasive by representatives from all of the countries participating in the UICC. Such data did not exist at the time the manual was being written.

In the current issue of the Journal, Rice and his colleagues ${ }^{4}$ at The Cleveland Clinic proposed a revised staging system for esophageal cancer. In terms more familiar to statisticians than to clinicians, their elegantly analyzed study examined the "monotonicity, distinctiveness, and homogeneity of survival in the context of current TNM classifications and stage groupings" based on a prospective 18-year database of 480 patients treated solely by esophageal resection. Otherwise stated, their analysis attempted to develop a staging system that reflects decreasing survival across increasing tumor stages, distinct differences in survival for each of the stage classifications, and no significant differences in survival among the TNM subsets included within each stage category. Rice and coworkers modeled their study on the pioneering approach used by Balch and colleagues 5 in analyzing a large international database to propose a new staging system for malignant melanoma-a system that has just been adopted by the AJCC and UICC in the sixth editions of the staging manuals. Balch's approach set a new standard for the development of evidencebased staging systems and has prompted similar efforts for other malignancies, including a study recently initiated by the International Association for the Study of Lung Cancer, aimed at achieving a comprehensive revision of the lung cancer staging system.

Relative to the current staging system, the salient features of the system proposed by Rice and coworkers include the following: the separation of T1 tumors into T1a (intramucosal) and T1b (submucosal) with T1a and high-grade dysplasia grouped together as stage I; a complete revision of the nodal descriptors based solely on the number of involved lymph nodes ( 0 to 2 vs 3 or more) rather than anatomic lymph 
node location; and recategorization of TNM subsets within just 4 stage classifications. The proposed change in the $\mathrm{T}$ descriptors appropriately reflects the increasing experience with very early cancers and our knowledge of the excellent long-term survival after resection of these tumors. The proposed revision of the $\mathrm{N}$ descriptors leads to a staging system that is conceptually analogous to the gastric cancer staging system, which ignores lymph node location and considers only the number of metastatic lymph nodes, with the caveat that at least 15 lymph nodes be removed for adequate staging. Achieving some concordance between the esophageal and gastric staging systems is important because the number of distal gastric cancers and proximal esophageal cancers is decreasing while the number of tumors arising in the area of the gastroesophageal junction is increasing. It is frequently very difficult to determine whether these tumors arise from the gastric or the esophageal side of the junction and which system should be used for staging. The current $\mathrm{N}$ descriptors in the esophageal staging system are also based on somewhat arbitrary definitions of what constitute "regional" (N1) nodes as opposed to more "distant" metastatic nodes (M1a). However, we know that lymph nodes metastases do not occur in this tightly defined anatomic manner in esophageal cancer. And, as pointed out by Rice and colleagues, the N1 versus M1a versus M1b descriptors do not accurately identify prognostically different groups.

Therefore, the study of Rice and coworkers is an important and very welcome addition to the literature on esophageal staging. It benefits from prospective data acquisition, a relatively large database, uniformity of patient treatment, lack of confounding factors (for example, use of induction therapy), and expert statistical analysis. The potential prognostic importance of the number of metastatic lymph nodes has been suggested by others previously. ${ }^{6}$ Additional studies from other institutions are needed to validate or modify the proposed staging system. The changes in the $\mathrm{T}$ descriptors are less likely to be controversial than those in the $\mathrm{N}$ and $\mathrm{M}$ descriptors. Questions remain: Are the categories of 0 to 2 versus 3 or more lymph nodes adequate? Is there a minimum number of lymph nodes or lymph node stations that should be examined to achieve adequate staging? Are there differences in this regard for the staging of distal esophageal versus gastroesophageal junction versus gastric cardia tumors? Should the M1 descriptor be reserved solely for distant visceral metastases? Ideally, the excellent work by Rice and colleagues will prompt other investigators to pursue similar studies that will ultimately allow a clinically meaningful revision of the esophageal staging system.

\section{References}

1. American Joint Committee on Cancer. AJCC Cancer Staging Handbook. 6th ed. Philadelphia: Lippincott-Raven; 2002.

2. Union Internationale Contre le Cancer. TNM classification of malignant tumours. 6th ed. New York: Wiley-Liss; 2002.

3. Casson AG, Rusch VW, Ginsberg RJ, Zankowicz N, Finley RJ. Lymph node mapping of esophageal cancer. Ann Thorac Surg. 1994; 58:1569-70.

4. Rice TW, Blackstone EH, Rybicki LA, Adelstein DJ, Murthy SC, DeCamp MM, et al. Refining esophageal cancer staging. $J$ Thorac Cardiovasc Surg. 2003;125;1103-13.

5. Balch CM, Buzaid AC, Atkins MB, Cascinelli N, Coit DG, Fleming ID, et al. A new American Joint Committee on Cancer staging system for cutaneous melanoma. Cancer. 2000;88:1484-91.

6. Korst RJ, Rusch VW, Venkatraman E, Bains MS, Burt ME, Downey RJ, et al. Proposed revision of the staging classification for esophageal cancer. J Thorac Cardiovasc Surg. 1998;115:660-70. 